\title{
Transfluthrin Eave-Positioned Targeted Insecticide (EPTI) Reduces Human Landing Rate of Pyrethroid Resistant and Susceptible Malaria Vectors in a Semi-Field Simulated Peridomestic Space
}

\author{
Mgeni Tambwe ( $\nabla$ mmohamed@ihi.or.tz ) \\ IHRDC: Ifakara Health Institute \\ Sarah J Moore \\ Ifakara Health Institute \\ Lorenz Hofer \\ Ifakara Health Institute \\ Ummi Abdul Kibondo \\ Ifakara Health Institute \\ Adam Saddler \\ Telethon Kids Institute,Perth,Australia
}

\section{Research}

Keywords: volatile pyrethroid, transfluthrin, pyrethroid resistance, eave-positioned targeted insecticide, EPTI, Anopheles gambiae s.S., Anopheles arabiensis, semi-field system

Posted Date: March 18th, 2021

DOI: https://doi.org/10.21203/rs.3.rs-310420/v1

License: (c) (7) This work is licensed under a Creative Commons Attribution 4.0 International License. Read Full License 


\section{Abstract}

Introduction: Volatile pyrethroids (VPs) are proven to reduce human-vector contact for mosquito vectors. With increasing resistance to pyrethroids in mosquitoes, the efficacy of VPs such as transfluthrin may be compromised. Therefore, experiments were conducted to determine if the efficacy of transfluthrin eave-positioned targeted insecticide (EPTI) depends on the resistance status of malaria vectors.

Methods: Ribbons treated with $5.25 \mathrm{~g}$ transfluthrin or untreated controls were used around the eaves of an experimental hut as EPTI inside a semi-field system. Mosquito strains with different levels of pyrethroid resistance were released simultaneously, recaptured by means of human landing catches (HLCs) conducted $2.5 \mathrm{~m}$ outside the hut, and monitored for 24-hour mortality. Technical-grade (TG) transfluthrin was used, followed by emulsifiable concentrate (EC) transfluthrin and additional mosquito strains. Generalised linear mixed models with binomial distribution were used to determine the impact of transfluthrin and mosquito strain on mosquito landing rates and 24-hour mortality.

Results: A significant interaction between strain and treatment indicated that the effect of the transfluthrin EPTI varied between three strains under investigation $(P<0.001)$. Whether TG or EC, EPTI significantly reduced the odds of landing of pyrethroid-susceptible mosquitoes Anopheles gambiae (Ifakara) and An. gambiae (Kisumu) and of pyrethroid-resistant mosquitoes An. arabiensis (Mbita), An. gambiae Kisumu knockdown-resistant (Kisumu-kdr) and An. arabiensis (Kingani), with PE $>40 \%$ for all strains $(P<0.001)$. In the control, An. gambiae mosquitoes were more likely to land than $A n$. arabiensis $(P<0.05)$.

Conclusions: This study confirms that the efficacy of EPTI was not dependent on mosquito pyrethroid resistance status. However, it remains unclear whether resistance to pyrethroids undermines the efficacy of transfluthrin for bite prevention. It is important to consider mosquito anthropophagy, strain, years of colonisation and fitness when assessing vector control interventions. Overall, these findings suggest that transfluthrin-treated EPTI could be useful in areas with highly pyrethroid-resistant mosquitoes. At this dosage, transfluthrin EPTI cannot be used to kill exposed mosquitoes.

\section{Introduction}

Indoor residual spraying (IRS) and long-lasting insecticidal nets (LLINs) are currently the core mosquito vector control tools employed in national malaria control programs worldwide [1]. Since 2000, global malaria incidence has decreased by $37 \%$ and mortality by $60 \%$ [2], to which these tools have contributed approximately $70 \%$ of the reduction [1]. However, there are concerns that progress has stagnated; the downward trend in malaria cases flattened and malaria increased in several countries between 2015 and 2019 [3]. Increased transmission in some areas where elimination was considered to be feasible has also been observed [4,5]. This increase is likely caused by insufficient coverage and use of core interventions, with fewer than half of households in sub-Saharan Africa owning enough nets for all occupants [3]. Progress may also be impeded by limitations of the core interventions and their effectiveness in certain settings. For example, the current tools do not provide complete protection outdoors in the peridomestic area, where humans and vectors frequently come into contact before bedtime [6]. Furthermore, the development of physiological resistance [7] in mosquito vectors may undermine the continued efficacy of IRS and LLINs [8].

Development of alternative control strategies that cover the existing gaps and that compliment core control tools remains necessary [9]. Proposed measures include spatial repellents (SR) [10,11], genetically engineered mosquitoes [12], attractive targeted (toxic) sugar bait (ATSB) [13] and endectocides such as ivermectin [14]. The focus of this study is SR from the pyrethroid class often referred to as volatile pyrethroids (VPs). VPs vaporise at room temperature and are dispersed into the surrounding area with the aim of creating a bite-free space [15], and they can be used indoors and outdoors. Previous studies have demonstrated that VPs such as transfluthrin and metofluthrin are effective at reducing the human landing rate (HLR) of a range of mosquitoes [16]. Passive emanators treated with transfluthrin or metofluthrin consistently demonstrated personal protective efficacy exceeding $50 \%$ in studies conducted in Cambodia [17], Tanzania [18], Belize [19] and Indonesia [20]. Transfluthrin applied to hessian strips as eave-positioned targeted insecticide (EPTI) has provided over $68 \%$ reduction in human vector contact in semi-field studies $[10,21]$ and over $80 \%$ in field studies in Tanzania [10, 11]. Volatile pyrethroids exhibit a dose response, with lower concentrations eliciting behavioural effects that include deterrence, excito-repellency and blood-feeding inhibition [22] and with higher concentrations or longer exposure times increasing knockdown and mortality [23].

Pyrethroid insecticides have been the main class of insecticide used in LLINs and IRS [24]. Resistance to these insecticides is now widespread [25], which poses a threat not only to the efficacy of LLINs and IRS but potentially also to VPs. Furthermore, effective, longlasting volatile insecticides of chemical classes other than pyrethroids are not yet available for public health use [26]. It is necessary to know whether the efficacy of VPs may be compromised by pyrethroid resistance and, therefore, if VPs can be used in areas with existing pyrethroid-resistant mosquito populations. VPs are from the same chemical class, which would normally indicate cross-resistance; however, 
structural differences between transfluthrin and non-volatile pyrethroid indicate that cross-resistance may not occur [27]. Therefore, the objectives of this study were to determine (1) the efficacy of transfluthrin applied as EPTI to reduce HLR of multiple strains of Afrotropical malaria vectors with varying levels of pyrethroid resistance and (2) delayed mortality induced by EPTI exposure.

\section{Methods}

\section{Study Site}

The experiment was conducted in a semi-field system (SFS) located in Bagamoyo, Tanzania, from March 2018 to October 2018 and from August 2019 to September 2019. The SFS measures $21 \times 29 \times 4.5 \mathrm{~m}$ and is divided into three compartments. Two heavy-duty polyethylene walls separate these compartments, preventing air movement between the chambers and reducing the chance of cross-contamination when working with VPs or other aerosols. The SFS allows for controlled experiments with disease-free mosquitoes to be conducted under field like climatic conditions [28]. In each compartment, an experimental hut [29] was constructed, and tests were conducted outside the huts to simulate a peridomestic space.

\section{Study Mosquitoes}

Five laboratory-reared mosquito strains were used in these experiments: (1) pyrethroid-susceptible Anopheles gambiae s.s. (Kisumu strain) and (2) An. gambiae s.s. (Kisumu-kdr strain) with L1014S kdr, i.e., kdr-east resistance mechanism [30], both originating from Kisumu, Kenya; (3) pyrethroid-susceptible An. gambiae s.s. (Ifakara strain) originating from Ifakara, Tanzania, and in colony at IHI since 1996; (4) pyrethroid-resistant An. arabiensis (Mbita strain) from the International Centre of Insect Physiology and Ecology (ICIPE), Kisumu, Kenya, expressing a moderate level of phenotypical resistance against permethrin and deltamethrin (the mechanism is likely metabolic but not confirmed); and (5) An. arabiensis (Kingani strain) originating from Ifakara and in colony at Bagamoyo since 2015, expressing a high level of phenotypical resistance against permethrin and deltamethrin [31]. The two An. arabiensis strains have been tested and found to be free of $k d r$ mutations (L1014F kdr-west and L1014S kdr-east) (unpublished data) commonly associated with pyrethroid resistance. It is likely that the metabolic resistance mechanism was responsible for their survival in the presence pyrethroid insecticides.

Before the start of semi-field experiments, susceptibility tests were conducted for each mosquito strain using tube test bioassays performed following World Health Organization (WHO) guidelines [32]. Non-blood-fed 3- to 5-day-old mosquitoes were exposed to insecticideimpregnated papers at the standard WHO discriminating dose for the pyrethroids permethrin $(0.75 \%)$ and deltamethrin $(0.05 \%)$. These insecticides were selected because they belong to the same chemical class as transfluthrin and are commonly used on LLINs.

All mosquito strains are maintained at the Bagamoyo branch of the Ifakara Health Institute (IHI) according to MR4 guidelines [33]. Larvae are fed on fish food (TetraMin $\circledast$ tropical flakes) and adult mosquitoes on $10 \%$ sucrose ad libitum. Bovine blood meals are provided to adult females for egg production using membrane-feeding assay. The insectary is maintained at $27 \pm 5^{\circ} \mathrm{C}$ and $70-100 \%$ relative humidity with approximately 12:12 light: dark (ambient lighting).

The experiments used 3- to 5-day-old female mosquitoes that had never blood-fed. The mosquitoes were sugar starved for 6 hours prior to the experiment. Because more than one mosquito strain with the same morphology was released simultaneously, red and yellow fluorescent pigments (Swada, Cheshire, UK) were used to differentiate between strains. Mosquitoes were marked in a cup by dusting the mesh lid of the cup with a brush containing the colour pigment; thereby creating a cloud of pigment that was transferred to the mosquitoes in small amounts. Preliminary experiments indicated that the fluorescent pigments did not influence mosquito survival or feeding behaviours.

\section{Preparation of transfluthrin eave-positioned targeted insecticide (EPTI)}

Hessian material has proved very useful for the delivery of transfluthrin because it has a much slower release rate than other textiles and thus increases the longevity of the VP device [21,34,35]. Hessian sacks were purchased locally, washed using well water and powder detergent (OMO ${ }^{8}$, Unilever, Nairobi, Kenya), dried under direct sunlight and then cut into $21 \mathrm{~m} \times 10 \mathrm{~cm}$ strips. The hessian was treated with either TG or EC transfluthrin formulations (Bayothrin EC, Bayer AG, Monheim am Rhein, Germany). The experiments were initially conducted using TG transfluthrin emulsified with $100 \mathrm{ml}$ of Tween ${ }^{20}$ (Sigma-Aldrich, CAS \#9005-64-5). Bayer developed and introduced EC transfluthrin that was used for further experiments. In all experiments, with either formulation, $5.25 \mathrm{~g}$ of transfluthrin was impregnated into hessian equivalent to $2.5 \mathrm{~g} / \mathrm{m}^{2}$. Drying took place out of direct sunlight to protect the transfluthrin from photolysis by exposure to ultraviolet light $[27,36]$. For the control arms, the strips were prepared in the same manner as the treated strips but with only water. During the day, the treated hessian was kept out of direct sunlight at the ambient outdoor temperature $\left(24-27.6^{\circ} \mathrm{C}\right)$ on a metal frame.

\section{Experimental procedure}


The primary aim of the study was to determine if pyrethroid resistance in mosquitoes has a negative impact on the efficacy of transfluthrin EPTI. To do this, the treated hessian was placed on the eaves gaps of experimental huts located in the SFS, out of direct sunlight (Fig. 1a). Applying insecticide in this targeted way exploits the natural movement of air rising inside houses and being funnelled out through the eaves, over the treated hessian and into the peridomestic space, helping to disperse insecticide.

Human landing catch (HLC) were conducted 2 m outside the experimental hut (Fig. 1b and c) to mimic the peridomestic environment. Mosquitoes were released outside the experimental hut at every corner of the SFS compartment, eliminating directional bias in their approach to the human volunteer. Three separate experiments were conducted to evaluate the efficacy of (1) TG transfluthrin EPTI against Ifakara strain, Mbita strain and Kingani strain mosquitoes; (2) EC transfluthrin EPTI against Ifakara strain, Mbita strain and Kingani strain mosquitoes; and (3) EC transfluthrin EPTI against Kisumu strain and Kisumu-kdr strain mosquitoes.

During each experiment, either transfluthrin EPTI or the control (water-treated hessian) was assigned to one of two separate compartments of the SFS. The treatments remained fixed for a block of four days, after which they were rotated. HLC volunteers rotated between compartments daily. Four volunteers were recruited but only two used each day. The experiment was conducted for 4 blocks over 16 days, after which each volunteer conducted HLC for each treatment 4 times in each compartment. The volunteers were rotated to control for any bias caused by individual attractiveness to mosquitoes [25]. Prior to the start of the experiment, for acclimatisation, mosquitoes were transferred from the insectary to the middle compartment of the SFS 30 min before their release.

Each day 80 mosquitoes of each strain were introduced into each compartment. Mosquitoes were separated into batches of 20 per strain and placed into 4 release cages, one in each corner of each compartment. The mosquitoes were released remotely by gently pulling strings connecting the release cages to simulate mosquitoes approaching the peridomestic space from multiple directions.

Throughout the experiment, volunteers wore shorts, covered shoes, and bug jackets to standardise the area available for mosquito landings. Mosquitoes that landed on the area between the ankle and the knee were collected using mouth aspirators through HLC (Fig. 1b).

Mosquitoes were recaptured continuously for 50 minutes every hour for 4 consecutive hours between 18:30 and 22:30 hrs. Each hour, a new collection cup was used and labelled with the time and date. These mosquitoes were transferred to the insectary after 4 hours, supplied with $10 \%$ sucrose and held for 24 hours to observe 24-hour mortality.

\section{Sample size}

Sample-size calculations were performed using simulation-based power analysis [25] in R statistical software version 3.02 (http://www.rproject.org) with a significance level of 0.05 for rejecting the null hypothesis. Data analysis for experimental data was planned to be conducted using generalised linear mixed models (GLMMs) [37]. Therefore, 1000 simulations of GLMMs approximating those used to analyse project data were run using a $2 \times 2$ Latin square design with volunteers rotating nightly. The power to predict the difference in mosquito landings between control and treatment was estimated as the proportion of the 1000 simulated data sets in which the null hypothesis was rejected when the GLMM was run. The simulations indicated that with an estimated 80 mosquitoes released per compartment per night and $60 \%$ recapture of released mosquitoes, there was $100 \%$ chance of detecting a $50 \%$ reduction in mosquito landings in the treatment arm after 16 nights of experimentation. Inter-observational variance among daily experiments was set at $5 \%$, and variability between times based on previous experiments was set at $25 \%$.

\section{Data Analysis}

Data were recorded on paper forms and double entered into Microsoft Excel. Cleaning and analysis were done in Stata 13 (StataCorp). For the WHO insecticide susceptibility tests, data were summarised as mean percentage (\%) 24-hour mortality of the four replicates and reported with $95 \%$ confidence intervals.

Data for each experiment using each transfluthrin formulation (EC or TG) were analysed separately.

The relative effect of transfluthrin on HLR and 24-hour mortality for different mosquito strains was investigated using GLMM with binomial distribution. For HLR, the dependent variable was the proportion of released mosquitoes that were recaptured. For mortality, the dependent variable was the recaptured proportion that died. Treatment, mosquito strain, compartment and volunteer were included as fixed categorical variables, with day included as a random effect. An interaction term between mosquito strain and treatment was included to determine if the effect of treatment varied between mosquito strains.

The protective efficacies of the transfluthrin EPTI against each mosquito strain were calculated as

Protective efficacy $(\mathrm{PE})=[(C-T) / C] \times 100 \%$, 
where $C$ stands for the number of mosquitoes landing in the control and $T$ for the number of mosquitoes landing in the treatment. The PE was calculated for each day, and the mean proportion of mosquitoes landing was reported with $95 \%$ confidence intervals (Cl). For 24 -hour mortality, the control-corrected mortality was calculated as

Control mortality $=(T-C) /(1-C) \times 100 \%$,

where $C$ and $T$ represents percentage mortality among mosquitoes landing in the control and treatment, respectively. The control-corrected mortality was calculated for each day, and the mean percentage dead was reported with $95 \% \mathrm{Cl}$.

\section{Results}

\section{WHO insecticide susceptibility tests}

The susceptibility status of each mosquito strain to permethrin and deltamethrin is presented in Table 1. An. gambiae Ifakara and Kisumu strains were fully susceptible. An. arabiensis Kingani, An. arabiensis Mbita and An. gambiae kdr were found to be resistant to pyrethroid. An. gambiae kdr was susceptible to deltamethrin.

Table 1

$\mathrm{KD}$ and 24-hour mortality of the malaria vectors tested during the WHO insecticide susceptibility test

\begin{tabular}{|c|c|c|c|}
\hline Mosquitoes & Insecticides & Concentration (\%) & 24-hour mortality* (\%) (95\% Cl) \\
\hline \multirow[t]{2}{*}{ Kisumu susceptible } & Permethrin & $(0.75)$ & $100(100-100)$ \\
\hline & Deltamethrin & $(0.05)$ & $100(100-100)$ \\
\hline \multirow[t]{2}{*}{ Kisumu-kdr } & Permethrin & $(0.75)$ & $98.9(95.8-100)$ \\
\hline & Deltamethrin & $(0.05)$ & $100(100-100)$ \\
\hline \multirow[t]{2}{*}{ Ifakara strain } & Permethrin & $(0.75)$ & $100(100-100)$ \\
\hline & Deltamethrin & $(0.05)$ & $100(100-100)$ \\
\hline \multirow[t]{2}{*}{ Mbita strain } & Permethrin & $(0.75$ & $72.6(59.9-87.9)$ \\
\hline & Deltamethrin & $(0.05)$ & $71.1(53.1-95.2)$ \\
\hline \multirow[t]{2}{*}{ Kingani strain } & Permethrin & $(0.75)$ & $19.7(10.1-38.6)$ \\
\hline & Deltamethrin & $(0.05)$ & $24.4(13.5-44.8)$ \\
\hline
\end{tabular}

\section{The efficacy of the transfluthrin EPTI against different mosquito strains}

In experiment 1 with TG transfluthrin, a significant interaction between strain and treatment was observed. This indicated that the effect of the transfluthrin EPTI varied between strains under investigation $(P<0.001$; Table 2). The use of TG transfluthrin EPTI significantly reduced the odds of landing of pyrethroid-susceptible An. gambiae (Ifakara strain; $\mathrm{OR}=0.22[0.18-0.26], P<0.001$ ) and had a similar impact on the landing of highly pyrethroid-resistant $A n$. arabiensis (Kingani; $\mathrm{OR}=0.23$ [0.19-0.27], $P<0.001$; Table 3). However, while the TG transfluthrin EPTI reduced the landing of pyrethroid-resistant An. arabiensis (Mbita), it did so to a lesser extent (OR $=0.33$ [0.28-0.39], $P<0.001$; Table 3). When assessing the efficacy of the EPTI using PE, the PE was similar for susceptible Ifakara 46.2 ( $95 \% \mathrm{Cl}: 45.6-65.5)$, moderately resistant Mbita 46.4 (95\% Cl: 37.9-54.9) and the highly resistant Kingani strain 54.9 (95\% Cl: 41.6-64.1; Table 3). The binomial GLMM for TG transfluthrin indicated that both volunteers 3 and 4 and compartment significantly influenced HLR (in both cases, $P<0.05$; Table 2).

Table 2. Generalised linear model output estimating the effect of EC/TG transfluthrin and mosquito strain on human landing rate in the semi-field system, Bagamoyo, Tanzania 


\begin{tabular}{|c|c|c|c|c|c|c|}
\hline \multirow[b]{2}{*}{ Variables } & \multicolumn{2}{|c|}{$\begin{array}{l}\text { Experiment 1, TG transfluthrin- } \\
(\underline{\underline{5.25} \mathrm{~g})}\end{array}$} & \multicolumn{2}{|c|}{$\begin{array}{l}\text { Experiment 2, EC transfluthrin } \\
(\underline{5.25} \mathrm{~g})\end{array}$} & \multicolumn{2}{|c|}{$\begin{array}{l}\text { Experiment } 3, \text { EC transfluthrin } \\
(\underline{\underline{5.25} \mathrm{~g})}\end{array}$} \\
\hline & $O R^{*}$ & $P$-value & $O R^{*}$ & $P$-value & $O R^{*}$ & $P$-value \\
\hline \multicolumn{7}{|l|}{ Treatment } \\
\hline Control & 1 & & 1 & & 1 & \\
\hline Transfluthrin & $0.22(0.18-0.26)$ & $<0.001$ & $0.10(0.08-0.12)$ & $<0.001$ & $0.14(0.12-0.17)$ & $<0.001$ \\
\hline \multicolumn{7}{|l|}{ Strain (In control) } \\
\hline Ifakara strain (Susceptible) & 1 & & 1 & & & \\
\hline Mbita strain (Metabolic) & $0.43(0.32-0.57)$ & $<0.001$ & $0.34(0.26-0.46)$ & $<0.001$ & - & - \\
\hline Kingani strain (Metabolic) & $0.60(0.45-0.80)$ & $<0.001$ & $0.44(0.33-0.59)$ & $<0.001$ & - & - \\
\hline Kisumu susceptible & - & - & - & - & 1 & \\
\hline Kisumu kdr & - & - & - & - & $1.0(0.86-1.17)$ & 0.05 \\
\hline \multicolumn{7}{|l|}{ Volunteers } \\
\hline Volunteer 1 & 1 & & 1 & & 1 & \\
\hline Volunteer 2 & $0.88(0.69-1.14)$ & 0.36 & $1.07(0.84-1.39)$ & 0.60 & $1.20(0.99-1.46)$ & 0.06 \\
\hline Volunteer 3 & $0.76(0.59-0.98)$ & 0.04 & $0.96(0.74-1.24)$ & 0.77 & $1.19(0.98-1.44)$ & 0.07 \\
\hline Volunteer 4 & $0.83(0.72-0.95)$ & 0.001 & $0.90(0.78-1.04)$ & 0.17 & $1.14(0.94-1.36)$ & 0.18 \\
\hline \multicolumn{7}{|l|}{ Compartment } \\
\hline Compart 1 & 1 & & 1 & & 1 & \\
\hline Compart 2 & $0.90(0.81-0.99)$ & 0.04 & $0.79(0.71-0.87)$ & $<0.001$ & $0.93(0.81-1.07)$ & 0.30 \\
\hline \multicolumn{7}{|l|}{ Treatment*strain } \\
\hline Transfluthrin*lfakara strain & 1 & & 1 & & & \\
\hline Transfluthrin*Mbita strain & $1.50(1.18-1.91)$ & $<0.001$ & $1.67(1.29--2.17)$ & $<0.001$ & - & - \\
\hline Transfluthrin*Kingani strain & $1.03(0.82-1.32)$ & 0.75 & $2.29(1.78-2.94)$ & $<0.001$ & - & - \\
\hline $\begin{array}{l}\text { Transfluthrin*Kisumu } \\
\text { susceptible }\end{array}$ & - & - & - & - & 1 & \\
\hline Transfluthrin*Kisumu kdr & - & - & - & - & $0.98(0.74-1.30)$ & 0.90 \\
\hline
\end{tabular}

* Odds ratio (OR) was adjusted for temperatures, humidity and all other variables in the table. 
Table 3

The adjusted odds ratio of mosquito landings and protective efficacy offered by EC and TG transfluthrin in the semi-field system, Bagamoyo, Tanzania

\begin{tabular}{|c|c|c|c|c|c|c|}
\hline \multirow{2}{*}{$\begin{array}{l}\text { Transfluthrin } \\
\text { EPTI }\end{array}$} & \multirow[t]{2}{*}{ Mosquitoes } & \multicolumn{2}{|c|}{ Landing in the presence EPTI } & \multicolumn{3}{|c|}{$\begin{array}{l}\text { Landing in the control } \\
\text { (reference) }\end{array}$} \\
\hline & & $\begin{array}{l}\mathrm{n}(\% \text { landing }[95 \% \\
\mathrm{Cl}])^{\mathrm{a}}\end{array}$ & OR $(95 \% \mathrm{Cl})$ & $\begin{array}{l}\mathrm{n}(\% \mathrm{l} \text { anding }[95 \% \\
\mathrm{Cl}])^{\mathrm{a}}\end{array}$ & $\begin{array}{l}\text { OR }(95 \% \\
\mathrm{Cl})\end{array}$ & $\begin{array}{l}\text { Protective efficacy (\% } \\
{[95 \% \text { Cl] })}\end{array}$ \\
\hline \multirow[t]{3}{*}{ TG } & Ifakara strain & $\begin{array}{l}500(39.0[32.9- \\
45.2])\end{array}$ & $\begin{array}{l}0.22(0.18- \\
0.26)^{\star}\end{array}$ & $\begin{array}{l}939(73.4[66.9- \\
79.8])\end{array}$ & 1 & $46.2(45.6-65.5)$ \\
\hline & $\begin{array}{l}\text { Mbita } \\
\text { arabiensis }\end{array}$ & $\begin{array}{l}370(29.5[24.4- \\
34.7])\end{array}$ & $\begin{array}{l}0.33(0.28- \\
0.39)^{\star}\end{array}$ & $\begin{array}{l}706(55.2[51.7- \\
58.6])\end{array}$ & 1 & $46.4(37.9-54.9)$ \\
\hline & Kingani strain & $\begin{array}{l}378(28.9[22.4- \\
35.4])\end{array}$ & $\begin{array}{l}0.23(0.19- \\
0.27)^{\star}\end{array}$ & $\begin{array}{l}804(62.8[56.4- \\
69.2])\end{array}$ & 1 & $54.9(41.6-64.1)$ \\
\hline \multirow[t]{3}{*}{ EC } & Ifakara strain & $\begin{array}{l}341(26.6[21.2- \\
32.1])\end{array}$ & $\begin{array}{l}0.17(0.14- \\
0.20)^{\star}\end{array}$ & $\begin{array}{l}980(76.6[70.3- \\
82.9])\end{array}$ & 1 & $65.0(57.0-72.2)$ \\
\hline & $\begin{array}{l}\text { Mbita } \\
\text { arabiensis }\end{array}$ & $\begin{array}{l}224(17.5[12.2- \\
22.8])\end{array}$ & $\begin{array}{l}0.23(0.19- \\
0.27)^{\star}\end{array}$ & $\begin{array}{l}697(54.5[51.9- \\
57.0])\end{array}$ & 1 & $67.6(57.6-77.6)$ \\
\hline & Kingani strain & $\begin{array}{l}347(27.1[20.5- \\
33.7])\end{array}$ & $\begin{array}{l}0.57 \\
(0.42--0.78)^{\star}\end{array}$ & $\begin{array}{l}774(60.5[56.6- \\
64.4])\end{array}$ & 1 & $55.6(45.6-65.5)$ \\
\hline \multirow[t]{2}{*}{ EC } & $\begin{array}{l}\text { Kisumu } \\
\text { susceptible }\end{array}$ & $\begin{array}{l}166(12.9[9.6- \\
16.3])\end{array}$ & $\begin{array}{l}0.14(0.11- \\
0.17)^{\star}\end{array}$ & $\begin{array}{l}647(50.5[50.0- \\
51.0])\end{array}$ & 1 & $74.3(67.7-80.9)$ \\
\hline & Kisumu kdr & $\begin{array}{l}164(12.8[9.6- \\
16.0])\end{array}$ & $\begin{array}{l}0.14(0.11- \\
0.17)^{\star}\end{array}$ & $\begin{array}{l}648(50.6[50.1- \\
51.1])\end{array}$ & 1 & $75.1(69.2-82.2)$ \\
\hline \multicolumn{7}{|c|}{$\begin{array}{l}\text { a Numbers in the control and treatment refer to the total number of mosquitoes caught/released during each experiment; the percenta } \\
\text { recaptured is in bracket. The percentage landing was calculated by dividing the number recaptured }(n) \text { by the total released }(N=1280) \\
\text { The OR is adjusted for temperature, humidity, compartment, volunteers and all other factors in the table. }\end{array}$} \\
\hline \multicolumn{7}{|c|}{ * $P$-value $<0.05$} \\
\hline
\end{tabular}

In experiment 2, using EC transfluthrin EPTI, there was again a significant interaction between strain and treatment, although a different trend was observed (Table 2). As with TG, the EC transfluthrin EPTI was observed to reduce the odds of landing of susceptible An. gambiae (Ifakara strain; OR $=0.17$ [0.14-0.20], $P<0.001$ ) and pyrethroid-resistant An. arabiensis (Mbita; OR =0.23 [0.19-0.27], $P<0.001$ ). However, EC transfluthrin showed lower efficacy against An. arabiensis (Kingani; OR $=0.57$ [0.42-0.78], $P<0.001$; Table 3). The model also indicated that compartment significantly influenced HLR of the mosquitoes (OR $=0.79$ [0.71-0.87], $P<0.001)$. None of the volunteers influenced HLR $(P>0.05$; Table 2).

Finally, in the analysis of the data from experiment 3, the interaction was not significant with Kisumu susceptible and kdr strains, indicating that the transfluthrin EPTI reduced landings of the two mosquitoes species in the same way (Table 2). The odds of landing of Kisumu susceptible and Kisumu kdr were equally reduced (OR = 0.14 [0.11-0.17], $P>0.001$; Table 3 ).

During the experiments, the average temperature was $27.8^{\circ} \mathrm{C}\left(23.8-31.5^{\circ} \mathrm{C}\right)$ and average relative humidity $(\mathrm{RH})$ was $76.5 \%(63.6-92 \%)$.

\section{Effect Of Species On HIr In The Control}

The effects of mosquito species on HLR were examined in the control. The two species colonised from wild mosquitoes in Ifakara, Tanzania, were compared. In both experiments, consistently higher catches were observed with the Ifakara strain than with the Kingani strain. For example, in experiment 2, An. gambiae s.s. (Ifakara) showed a higher landing proportion, with an average of $76.6 \%$ (95\% $\mathrm{Cl}$ : 70.3-82.9), than did An. arabiensis (Kingani), with an average of $60.5 \%$ (95\% Cl: $56.6-64.4)$, and this difference was significant (OR $=0.5$ [95\% Cl: 0.4-0.6], $P<0.001$; Table 4). 
Table 4

The adjusted odds ratio of mosquito landings and protective efficacy offered by EC and TG transfluthrin in the semi-field system, Bagamoyo, Tanzania

\begin{tabular}{|c|c|c|c|c|c|}
\hline \multirow{2}{*}{$\begin{array}{l}\text { Transfluthrin } \\
\text { EPTI }\end{array}$} & \multirow[t]{2}{*}{ Mosquitoes } & \multicolumn{2}{|c|}{ Landing in the presence of EPTI } & \multicolumn{2}{|l|}{ Landing in the control } \\
\hline & & n (\% landing $[95 \% \mathrm{Cl}])^{a}$ & OR $(95 \% \mathrm{Cl})$ & n (\% landing $[95 \% \mathrm{Cl}])^{a}$ & OR $(95 \% \mathrm{Cl})$ \\
\hline \multirow[t]{3}{*}{ TG } & Ifakara strain & $500(39.0$ [32.9-45.2]) & 1 & 939, (73.4 [66.9-79.8]) & 1 \\
\hline & Mbita arabiensis & 370 (29.5 [24.4-34.7]) & $0.65(0.49-0.86)^{\star}$ & 706 (55.2 [51.7-58.6]) & $0.43(0.32-0.57)^{\star}$ \\
\hline & Kingani strain & 378 (28.9 [22.4-35.4]) & $0.62(0.47-0.83)^{*}$ & $804(62.8$ [56.4-69.2]) & $0.60(0.45-0.80) *$ \\
\hline \multirow[t]{3}{*}{$\mathrm{EC}$} & Ifakara strain & 341 (26.6 [21.2-32.1]) & 1 & $980(76.6[70.3-82.9)$ & 1 \\
\hline & Mbita arabiensis & 224 (17.5 [12.2-22.8]) & $0.58(0.43-0.78)^{\star}$ & $697(54.5[51.9-57.0])$ & $0.34(0.25-0.46) *$ \\
\hline & Kingani strain & 347 (27.1 [20.5-33.7]) & $1.01(0.76-1.38)$ & $774(60.5[56.6-64.4])$ & $0.44(0.33-0.59)$ * \\
\hline \multirow[t]{2}{*}{ EC } & Kisumu susceptible & $166(12.9[9.6-16.3])$ & 1 & $647(50.5[50.0-51.0])$ & 1 \\
\hline & Kisumu kdr & $164(12.8[9.6-16.0])$ & $0.99(0.78-1.24)$ & $648(50.6[50.1-51.1])$ & $1.00(0.86-1.17)$ \\
\hline
\end{tabular}

a Numbers in the control and treatment refer to the total number of mosquitoes caught/released during each experiment; the percentage recaptured is in bracket. The percentage landing was calculated by dividing the number recaptured $(n)$ by the total released $(N=1280)$. The ORs are adjusted for temperature, humidity, compartment, volunteers and all other factors in the table. * $P$-value $<0.05$

Comparison of 24-hour mortality induced by transfluthrin-treated eave ribbon between mosquito strains.

At $5.25 \mathrm{~g}$ dosage, no significant difference in 24-hour mortality was observed in the presence of transfluthrin EPTI compared to the control across all mosquitoes strains $(P>0.05)$.

\section{Discussion}

\section{The efficacy of EPTI to reduce HLR of malaria vectors}

This study was conducted to determine if pyrethroid resistance in mosquitoes would have a negative impact on the efficacy of transfluthrin EPTI. Findings showed that An. arabiensis Kingani strain mosquitoes expressing high phenotypical resistance to pyrethroids were less repelled than the moderately resistant Mbita strain when using EC transfluthrin. However, Kingani, Mbita and Ifakara strains were equally repelled when using TG transfluthrin. It is therefore unclear how the different levels of metabolic resistance affect the efficacy of transfluthrin EPTI. TG was less effective against Mbita than against the susceptible Ifakara strain (An. gambiae), while EC was less effective against both the Mbita and the Kingani strains (An. arabiensis). This may indicate that metabolic resistance is indeed detrimental to the efficacy of transfluthrin; however, it is important to be cautioned when comparing species that have different levels of human biting preference (An. gambiae, An. arabiensis) because it is unknown how this variation affects the efficacy of transfluthrin. This study used An. gambiae s.s as a reference strain because colonisation of the susceptible An. arabiensis strain was not possible due to widespread resistance.

Our results suggest that $k d r$ target site mutations do not reduce the efficacy of transfluthrin. However, this finding must be interpreted with caution because the susceptibility test of the mosquitoes used revealed low levels of phenotypic resistance. What is clear from this study is that, compared to the control, transfluthrin EPTI can reduce landings of resistant mosquitoes. These findings corroborate previous experiments conducted under field settings in Kilombero Valley, Tanzania [10, 11, 38], in which transfluthrin applied to hessian in eaves (at concentrations higher than $5.25 \mathrm{~g}$ ) significantly reduced HLR by over $80 \%$ and as well in the SFS, where the PE was over $68 \%$ [39]. Andres et al. observed that transfluthrin-treated polyester strips provide significant protection in the semi-field using one species of mosquito that was moderately resistant to pyrethroid [39]. Furthermore, transfluthrin-treated eave ribbon provided protection in Kilombero Valley, where malaria transmission is transmitted by An.arabiensis and An. funestus mosquitoes [40], which were confirmed to be highly resistant to pyrethroid [31]. Methodologies used by these previous experiments were not designed to directly compare the differences in HLR between pyrethroidsusceptible and resistant mosquitoes. This study, however, provides a unique opportunity to compare the efficacy of transfluthrin applied as EPTI across different mosquito strains expressing different types and levels of insecticide resistance. Much more work is needed in this area, looking at a wider range of mosquito strains and resistance mechanisms.

It is known that the structural differences between VPs. such as transfluthrin, which contain tetrafluorobenzyl alcohol, and non-VPs, such as permethrin, which contain phenoxybenzyl alcohol, may explain the efficacy of transfluthrin against resistant mosquitoes [41]. Hortsman et 
al. observed that the enzyme responsible for detoxification of non-VPs is unable to bind to the tetrafluorobenzyl moiety of VPs, leaving them active against resistant mosquitoes [27]. Further work is needed to determine the mechanism that causes mosquitoes to be repelled by transfluthrin in order to ascertain whether cross-resistance is possible. On the other hand, combining multiple active ingredients in targeted eave applications may help to combat resistant mosquitoes. Strategies could also combine an SR with a chemical that has high-contact toxicity and thus kills those mosquitoes that are not repelled and that are attempting to enter through the eaves. It was observed that mosquitoes attempting to enter houses spend $80 \%$ of their time within $30 \mathrm{~cm}$ of the eave [42]; thus, adding a second Al may enhance the control of resistant vectors. As has beenlt was observed in one study that the addition of the synergist piperonyl butoxide (PBO) can enhance knockdown by mosquito coils treated with a VP [43].

Despite transfluthrin's reduction of the HRL, inconsistent findings were observed when using PE for measuring efficacy compared to the OR estimates from the model. Such difference may be because OR from the GLMM contains additional explanatory variables that are not considered in calculating the PE. It is therefore suggested that for the evaluation of spatial repellent in the semi-field system, GLMM estimates should be presented rather than the calculated PE. The GLMM estimates are more robust as they account for other variables.

\section{The effect of transfluthrin formulation on HLR}

While the EC and TG formulations were not compared directly, the EC did produce higher reductions in HLR. This could be explained by formulation differences that may have resulted in higher release rates and thus in different amounts of transfluthrin available in the air. It is known that differential concentrations of transfluthrin will induce different behaviours, including avoidance, irritancy, knockdown and mortality [44]. This dosage-dependent difference in mosquito behavioural response is also observed in other pyrethroid insecticides, including deltamethrin, cyphenothrin, d-tetramethrin and tetramethrin [45]. The practical advantage of using EC was that it readily dissolves in water, making it more convenient to use, whereas TG transfluthrin required emulsification with detergent to mix with water. Further investigation into transfluthrin formulations is needed to fully assess their efficacy.

\section{The influence of species and strain on HLR}

In addition to resistance, mosquito landing (HLR) was likely to be influenced by other factors (Fig. 2). In the absence of transfluthrin, this study observed differences in landing for the two different mosquito species. The Ifakara strain (An. gambiae) had a higher proportion of landing than did the Kingani strain (An. arabiensis) or the Mbita strain (An. arabiensis). Despite having been colonised for more than 10 years on particular Ifakara and Kingani strains, these mosquitoes demonstrated a behaviour seen in wild mosquitoes. Gilles et al. conducted an experiment in the field where they observed that An. gambiaes.s. were more likely that An. arabiensis strains to land on the person conducting HLC, indicating that species differences influence mosquito landing $[46,47]$. The differences in landing between these mosquito species is caused by differences in attraction to human cues [46]. An. arabiensis feed on both human and animals [48] depending on the relative abundance [49] or availability [50] of humans and animals, whereas An. gambiae s.s. feed exclusively on humans [51]. It is therefore suggested that the anthropophilic behaviour of An. gambiaes.s. may influence landing of these mosquitoes compared to the more opportunistic An. arabiensis.

Furthermore, the response of different species to VPs is well documented, with higher doses of transfluthrin needed to elicit escape responses in robust species such as Aedes aegypti than in Anopheles mosquitoes [44] and with different responses of members of the An. minimus complex to pyrethroids and DDT [52]. It is also known that species vary in their sensitivity to topical repellents [53]. Therefore, in evaluating the efficacy of volatile pyrethroids, it is important to investigate the species and strains that will ultimately be targeted.

The difference in behavioural response of mosquitoes in the presence of repellent may also be associated with age. Studies have demonstrated that younger mosquitoes showed lower response to topical mosquito repellents [54], with very old mosquitoes being more responsive to repellents [55]. This study followed WHO guidance, using younger mosquitoes that are less likely to be affected by pyrethroid exposure [56]. Because the use of young mosquitoes may underestimate the PE of the VP, it is therefore recommended that further work be carried out on the optimal physiological age of mosquitoes to be used in studies of VP.

\section{4-hour mortality of malaria vectors after exposure to transfluthrin}

The transfluthrin dose used in this study did not induce mortality for any of the mosquito strains; therefore, we were unable to determine if there was cross-resistance between traditional pyrethroids and transfluthrin. Exposure to doses above $5.25 \mathrm{~g}$ of transfluthrin and long exposure have been associated with increased mortality in exposed mosquitoes [22, 57], so these higher doses would be required to determine if there is any difference between resistant and susceptible strains. Only those mosquitoes that were recaptured by HLC were examined for 24-hour mortality; therefore, the full impact of transfluthrin on mortality cannot be measured. It is possible that those that did not land may have received a higher and potentially more lethal dose of transfluthrin. While it is useful to know if a mosquito will survive 
after a bite (and thus potentially go on to transmit disease), a better picture of the efficacy of VPs would be achieved if all mosquitoes were accounted for.

\section{Conclusion}

Transfluthrin EPTI offered protection against all mosquito species regardless of the mosquitoes' level of resistance. However, the differences in effect observed in different mosquitoes species highlight the fact that resistance in mosquitoes may be detrimental to the efficacy of transfluthrin. These findings demonstrated that transfluthrin-treated EPTI could be used to control malaria in areas with pyrethroid-resistant mosquitoes. Although this study suggests that EPTI reduces human landing rate for both mosquitoes, additional evidence is needed to determine whether resistance in mosquitoes is detrimental to the efficacy of transfluthrin. This is particularly important in areas where transfluthrin will be considered for the control of mosquito vectors [20].

\section{Declarations}

Ethics approval and consent to participate

Permission to conduct these experiments was granted by ethical review committees at Ifakara Health Institute (IHI/IRB/No: 024-2016) and the National Institute for Medical Research (NIMR/HQ/R.8a/Vol.IX/2381). The volunteers participating in these experiments were IHI employees skilled in performing HLC. They were recruited voluntarily with written informed consent after the risks and benefits of the study procedures and their right to leave at any time during the study was clearly explained. All mosquitoes used in this experiment were laboratory-reared with low risk of transmitting malaria parasite.

\section{Consent for publication}

The Director General of NIMR granted the permission to publish this work.

Availability of data and materials

Data generated and analysed for this study are included in this article and its supplementary information files. (Additional file 1)

\section{Competing interests}

The authors declare that they have no competing interests. SJM and UAK conduct contract product evaluation of a number of vector control tools.

\section{Funding}

Ifakara Health Institute Vector Control Product Testing Unit (VCPTU) covered experimental costs. Salaries for Mgeni Tambwe, Sarah Moore and Adam Saddler were funded through a grant from the Innovative Vector Control Consortium (IVCC). IVCC would like to acknowledge that source funding for the 'Push-Pull' project came from the Bill \& Melinda Gates Foundation and UK Aid.

\section{Authors' contributions}

SJM, AS and MMT conceived the study; AS and MMT performed the data collection; LH performed the molecular susceptibility assay for the An. arabiensis mosquitoes; AS, SJM, MMT and UAK performed data analysis; MMT wrote the manuscript; AS and SJM revised the manuscript. SJM and AS critically revised the final draft. All authors revised the final draft.

\section{Acknowledgements}

Thanks to Bayer AG for generously donated the transfluthrin used in these experiments.

\section{References}

1. Bhatt S, Weiss DJ, Cameron E, Bisanzio D, Mappin B, Dalrymple U, Battle K, Moyes CL, Henry A, Eckhoff PA, et al. The effect of malaria control on Plasmodium falciparum in Africa between 2000 and 2015. Nature. 2015;526:207-11.

2. Cibulskis RE, Alonso P, Aponte J, Aregawi M, Barrette A, Bergeron L, Fergus CA, Knox T, Lynch M, Patouillard E, et al. Malaria: global progress 2000-2015 and future challenges. Infect Dis Poverty. 2016;5:61.

3. World Health Organization (WHO). The world malaria report. Geneva: WHO; 2020. 
4. Temu EA, Maxwell C, Munyekenye G, Howard AF, Munga S, Avicor SW, Poupardin R, Jones JJ, Allan R, Kleinschmidt I, et al. Pyrethroid resistance in Anopheles gambiae, in Bomi County, Liberia, compromises malaria vector control. PLoS One. 2012;7:e44986.

5. Reddy MR, Overgaard HJ, Abaga S, Reddy VP, Caccone A, Kiszewski AE, Slotman MA. Outdoor host seeking behaviour of Anopheles gambiae mosquitoes following initiation of malaria vector control on Bioko Island, Equatorial Guinea. Malar J. 2011;10:184.

6. Monroe A, Moore S, Koenker H, Lynch M, Ricotta E. Measuring and characterizing night time human behaviour as it relates to residual malaria transmission in sub-Saharan Africa: a review of the published literature. Malar J. 2019;18:6.

7. Hancock PA, Wiebe A, Gleave KA, Bhatt S, Cameron E, Trett A, Weetman D, Smith DL, Hemingway J, Coleman M, et al. Associated patterns of insecticide resistance in field populations of malaria vectors across Africa. Proc Natl Acad Sci U S A. 2018;115:5938-43.

8. Sougoufara S, Doucouré S, Sembéne PMB, Harry M, Sokhna C. Challenges for malaria vector control in sub-Saharan Africa: resistance and behavioral adaptations in Anopheles populations. J Vector Borne Dis. 2017;54:4-15.

9. Sougoufara S, Ottih EC, Tripet F. The need for new vector control approaches targeting outdoor biting anopheline malaria vector communities. Parasit Vectors. 2020;13:295.

10. Ogoma SB, Mmando AS, Swai JK, Horstmann S, Malone D, Killeen GF. A low technology emanator treated with the volatile pyrethroid transfluthrin confers long term protection against outdoor biting vectors of lymphatic filariasis, arboviruses and malaria. PLoS Negl Trop Dis. 2017;11:e0005455.

11. Masalu JP, Finda M, Okumu FO, Minja EG, Mmbando AS, Sikulu-Lord MT, Ogoma SB. Efficacy and user acceptability of transfluthrintreated sisal and hessian decorations for protecting against mosquito bites in outdoor bars. Parasit Vectors. 2017;10:197.

12. Benelli G, Jeffries CL, Walker T. Biological control of mosquito vectors: past, present, and future. Insects. $2016 ; 7(4): 52$.

13. Marshall JM, White MT, Ghani AC, Schlein Y, Muller GC, Beier JC. Quantifying the mosquito's sweet tooth: modelling the effectiveness of attractive toxic sugar baits (ATSB) for malaria vector control. Malar J. 2013;12:291.

14. Chaccour C, Barrio A, Gil Royo AG, Martinez Urbistondo D, Slater H, Hammann F, Del Pozo JL. Screening for an ivermectin slow-release formulation suitable for malaria vector control. Malar J. 2015;14:102.

15. Achee NL, Bangs MJ, Farlow R, Killeen GF, Lindsay S, Logan JG, Moore SJ, Rowland M, Sweeney K, Torr SJ, et al. Spatial repellents: from discovery and development to evidence-based validation. Malar J. 2012;11:164.

16. Bibbs CS, Kaufman PE. Volatile pyrethroids as a potential mosquito abatement tool: a review of pyrethroid-containing spatial repellents. J Integr Pest Manag 2017;8(1).

17. Liverani M, Charlwood JD, Lawford H, Yeung S. Field assessment of a novel spatial repellent for malaria control: a feasibility and acceptability study in Mondulkiri, Cambodia. Malar J. 2017;16:412.

18. Kawada H, Temu EA, Minjas J, Matsumoto O, Iwasaki T, Takagi M. Field evaluation of spatial repellency of metofluthrin-impregnated plastic strips against Anopheles gambiae complex in Bagamoyo, coastal Tanzania. J Am Mosq Control Assoc. 2008;24:404-9.

19. Wagman JM, Grieco JP, Bautista K, Polanco J, Briceno I, King R, Achee NL. The field evaluation of a push-pull system to control malaria vectors in northern Belize, Central America. Malar J. 2015;14:184.

20. Syafruddin D, Asih PBS, Rozi IE, Permana DH, Nur Hidayati AP, Syahrani L, Zubaidah S, Sidik D, Bangs MJ, Bøgh C, et al. Efficacy of a spatial repellent for control of malaria in Indonesia: a cluster-randomized controlled trial. Am J Trop Med Hyg. 2020;103:344-58.

21. Ogoma SB, Ngonyani H, Simfukwe ET, Mseka A, Moore J, Maia MF, Moore SJ, Lorenz LM. The mode of action of spatial repellents and their impact on vectorial capacity of Anopheles gambiae sensu stricto. PLoS One. 2014;9:e110433.

22. Ogoma SB, Lorenz LM, Ngonyani H, Sangusangu R, Kitumbukile M, Kilalangongono M, Simfukwe ET, Mseka A, Mbeyela E, Roman D, et al. An experimental hut study to quantify the effect of DDT and airborne pyrethroids on entomological parameters of malaria transmission. Malar J. 2014;13:131.

23. Ten Bosch QA, Castro-Llanos F, Manda H, Morrison AC, Grieco JP, Achee NL, Perkins TA. Model-based analysis of experimental data from interconnected, row-configured huts elucidates multifaceted effects of a volatile chemical on Aedes aegypti mosquitoes. Parasit Vectors. 2018;11:365.

24. Zaim M, Aitio A, Nakashima N. Safety of pyrethroid-treated mosquito nets. Med Vet Entomol. 2000;14:1-5.

25. Mitchell SN, Stevenson BJ, Muller P, Wilding CS, Egyir-Yawson A, Field SG, Hemingway J, Paine MJ, Ranson H, Donnelly MJ. Identification and validation of a gene causing cross-resistance between insecticide classes in Anopheles gambiae from Ghana. Proc Natl Acad Sci U S A. 2012;109:6147-52.

26. Norris EJ, Coats JR. Current and future repellent technologies: the potential of spatial repellents and their place in mosquito-borne disease control. Int J Environ Res Public Health. 2017;14(2):124. 
27. Horstmann S, Sonneck R. Contact bioassays with phenoxybenzyl and tetrafluorobenzyl pyrethroids against target-site and metabolic resistant mosquitoes. PLoS One. 2016;11:e0149738.

28. Ferguson HM, Ng'habi KR, Walder T, Kadungula D, Moore SJ, Lyimo I, Russell TL, Urassa H, Mshinda H, Killeen GF, Knols BG. Establishment of a large semi-field system for experimental study of African malaria vector ecology and control in Tanzania. Malar J. 2008;7:158.

29. Okumu FO, Moore J, Mbeyela E, Sherlock M, Sangusangu R, Ligamba G, Russell T, Moore SJ. A modified experimental hut design for studying responses of disease-transmitting mosquitoes to indoor interventions: the Ifakara experimental huts. PLoS One. 2012;7:e30967.

30. Stump AD, Atieli FK, Vulule JM, Besansky NJ. Dynamics of the pyrethroid knockdown resistance allele in western Kenyan populations of Anopheles gambiae in response to insecticide-treated bed net trials. Am J Trop Med Hyg. 2004;70:591-6.

31. Matowo NS, Munhenga G, Tanner M, Coetzee M, Feringa WF, Ngowo HS, Koekemoer LL, Okumu FO. Fine-scale spatial and temporal heterogeneities in insecticide resistance profiles of the malaria vector, Anopheles arabiensis in rural south-eastern Tanzania. Wellcome Open Res. 2017;2:96.

32. World Health Organization (WHO). Test procedure for insecticide resistance monitoring in malaria vector mosquitoes. 2nd ed. WHO. 2018. https://apps.who.int/iris/bitstream/handle/10665/250677/9789241511575-eng.pdf?sequence=9789241511571.

33. MR4. Methods in Anopheles research manual. Malaria Research and Reference Reagent Resource Centre; 2016. Accessed on 07.03.2021 https://www.beiresources.org/Portals/2/VectorResources/2016\%20Methods\%20in\%20Anopheles\%20Research\%20full\%20manual.pdf.

34. Mmbando AS, Ngowo H, Limwagu A, Kilalangongono M, Kifungo K, Okumu FO. Eave ribbons treated with the spatial repellent, transfluthrin, can effectively protect against indoor-biting and outdoor-biting malaria mosquitoes. Malar J. 2018;17:368.

35. Mmbando AS, Batista EPA, Kilalangongono M, Finda MF, Mwanga EP, Kaindoa EW, Kifungo K, Njalambaha RM, Ngowo HS, Eiras AE, Okumu FO. Evaluation of a push-pull system consisting of transfluthrin-treated eave ribbons and odour-baited traps for control of indoor- and outdoor-biting malaria vectors. Malar J. 2019;18:87.

36. World Health Organization (WHO). Transfluthrin. (WHO specification and evaluations for public health pesticides). WHO: n.d.

37. Bates D, Mächler M, Bolker B, Walker S. Fitting linear mixed-effects models Using Ime4. J Stat Softw 2015;67(1).

38. Mmbando AS, Ngowo HS, Kilalangongono M, Abbas S, Matowo NS, Moore SJ, Okumu FO. Small-scale field evaluation of push-pull system against early- and outdoor-biting malaria mosquitoes in an area of high pyrethroid resistance in Tanzania. Wellcome Open Res. 2017;2:112.

39. Andrés M, Lorenz LM, Mbeleya E, Moore SJ. Modified mosquito landing boxes dispensing transfluthrin provide effective protection against Anopheles arabiensis mosquitoes under simulated outdoor conditions in a semi-field system. Malar J. 2015;14:255.

40. Lwetoijera DW, Harris C, Kiware SS, Dongus S, Devine GJ, McCall PJ, Silas Majambere S. Increasing role of Anopheles funestus and Anopheles arabiensis in malaria transmission in the Kilombero Valley, Tanzania. Malar J. 2014;13:331.

41. Bohbot JD, Fu L, LE TC, Chauhan KR, Cantrell CL, Dickens JC. Multiple activities of insect repellents on odorant receptors in mosquitoes. Med Vet Entomol. 2011;25:436-44.

42. Spitzen J, Koelewijn T, Mukabana WR, Takken W. Visualization of house-entry behaviour of malaria mosquitoes. Malar J. 2016;15:110.

43. Katsuda Y, Leemingsawat S, Thongrungkiat S, Komalamisara N, Kanzaki T, Watanabe T, Kahara T. Control of mosquito vectors of tropical infectious diseases: (1) bioefficacy of mosquito coils containing several pyrethroids and a synergist. Southeast Asian J Trop Med Public Health. 2008;39:48-54.

44. Sukkanon C, Nararak J, Bangs MJ, Hii J, Chareonviriyaphap T. Behavioral responses to transfluthrin by Aedes aegypti, Anopheles minimus, Anopheles harrisoni, and Anopheles dirus (Diptera: Culicidae). PLoS One. 2020;15:e0237353.

45. Mongkalangoon P, Grieco JP, Achee NL, Suwonkerd W, Chareonviriyaphap T. Irritability and repellency of synthetic pyrethroids on an Aedes aegypti population from Thailand. J Vector Ecol. 2009;34:217-24.

46. Gillies MT. Selection for host preference in Anopehles gambiae. Nature. 1964;203:852-4.

47. Curtis CF, Lines Jd, ljumba J, Callaghan A, Hill N, Karimzad MA. The relative efficacy of repellents against mosquito vectors of disease. Med Vet Entomol. 1987;1:109-19.

48. Mahande A, Mosha F, Mahande J, Kweka E. Feeding and resting behaviour of malaria vector, Anopheles arabiensis with reference to zooprophylaxis. Malar J. 2007;6:100. 
49. Asale A, Duchateau L, Devleesschauwer B, Huisman G, Yewhalaw D. Zooprophylaxis as a control strategy for malaria caused by the vector Anopheles arabiensis (Diptera: Culicidae): a systematic review. Infect Dis Poverty. 2017;6:160.

50. Iwashita H, Dida GO, Sonye GO, Sunahara T, Futami K, Njenga SM, Chaves LF, Minakawa N. Push by a net, pull by a cow: can zooprophylaxis enhance the impact of insecticide treated bed nets on malaria control? Parasit Vectors. 2014;7:52.

51. Costantini C, Sagnon N, della Torre A, Coluzzi M. Mosquito behavioural aspects of vector-human interactions in the Anopheles gambiae complex. Parassitologia. 1999;41:209-17.

52. Potikasikorn J, Chareonviriyaphap T, Bangs MJ, Prabaripai A. Behavioral responses to DDT and pyrethroids between Anopheles minimus species A and C, malaria vectors in Thailand. Am J Trop Med Hyg. 2005;73:343-9.

53. Van Roey K, Sokny M, Denis L, Van den Broeck N, Heng S, Siv S, Sluydts V, Sochantha T, Coosemans M, Durnez L. Field evaluation of picaridin repellents reveals differences in repellent sensitivity between southeast Asian vectors of malaria and arboviruses. PLos Negl Trop Dis. 2014;8:e3326.

54. Xue RD, Barnard DR. Human host avidity in Aedes albopictus: influence of mosquito body size, age, parity, and time of day. J Am Mosq Control Assoc. 1996;12(1):58-63.

55. Mulatier M, Porciani A, Nadalin L, Ahoua Alou LP, Chandre F, Pennetier C, Dormont L, Cohuet A. DEET efficacy increases with age in the vector mosquitoes Anopheles gambiae s.s. and Aedes albopictus (Diptera: Culicidae). J Med Entomol. 2018;55:1542-8.

56. Aldridge RL, Kaufman PE, Bloomquist JR, Gezan SA, Linthicum KJ. Application site and mosquito age influences malathion- and permethrin-induced mortality in Culex quinquefasciatus (Diptera: Culicidae). J Med Entomol. 2017;54:1692-8.

57. Chadwick PR. The activity of some pyrethroids, DDT and lindane in smoke from coils for biting inhibition, knockdown and kill of mosquitoes (Diptera, Culicidae). Bull Ent Res. 1975;65:97-107.

\section{Figures}



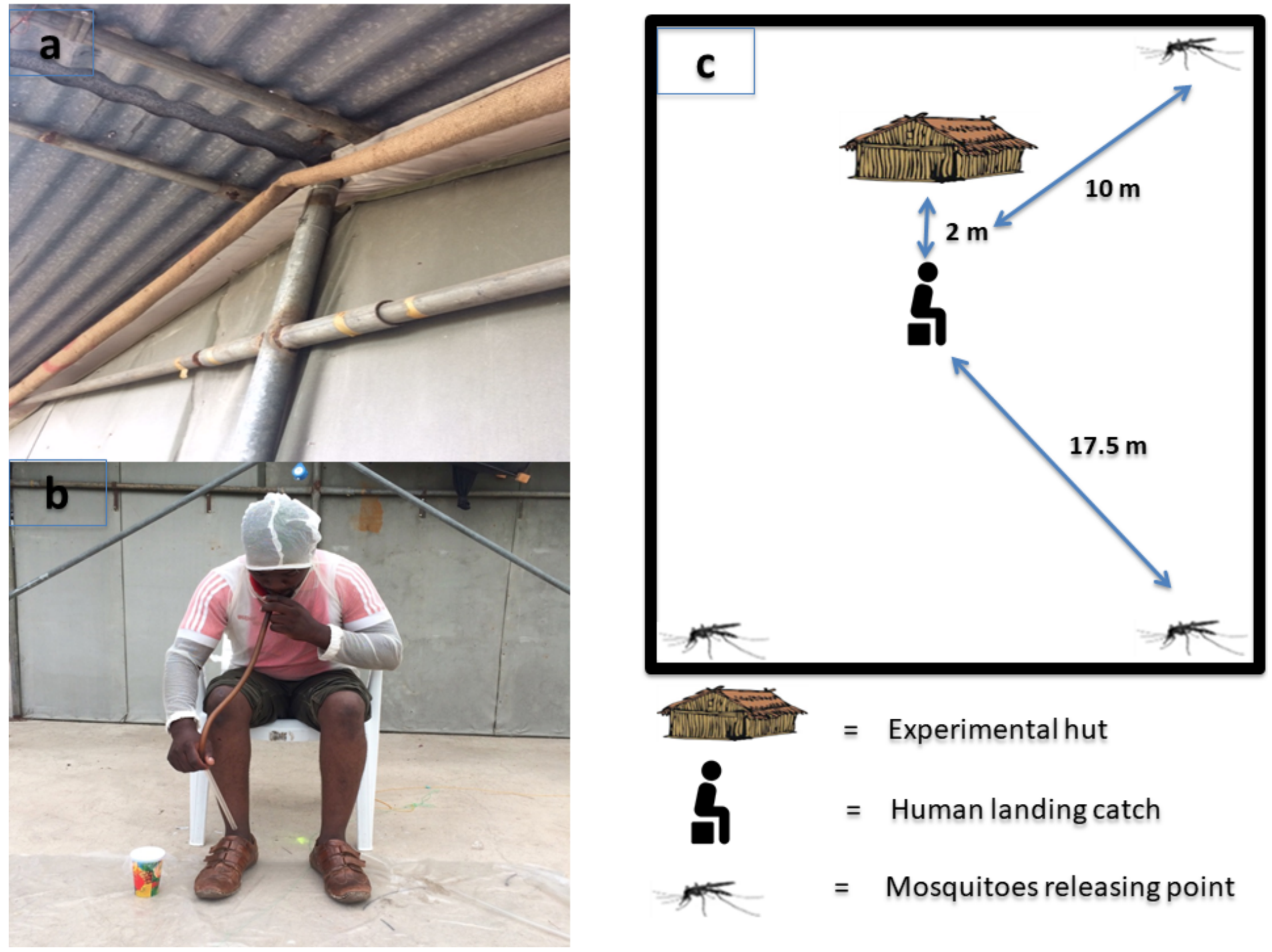

Figure 1

The evaluation of transfluthrin EPTI in the semi-field system (a) Yellowish strips represent transfluthrin hessian strip position on the eave " EPTI" (b) A volunteer sitting outside the experiment hut conducting HLC (c) The schematic representation of the experiment inside a compartment of the semi-field system. 


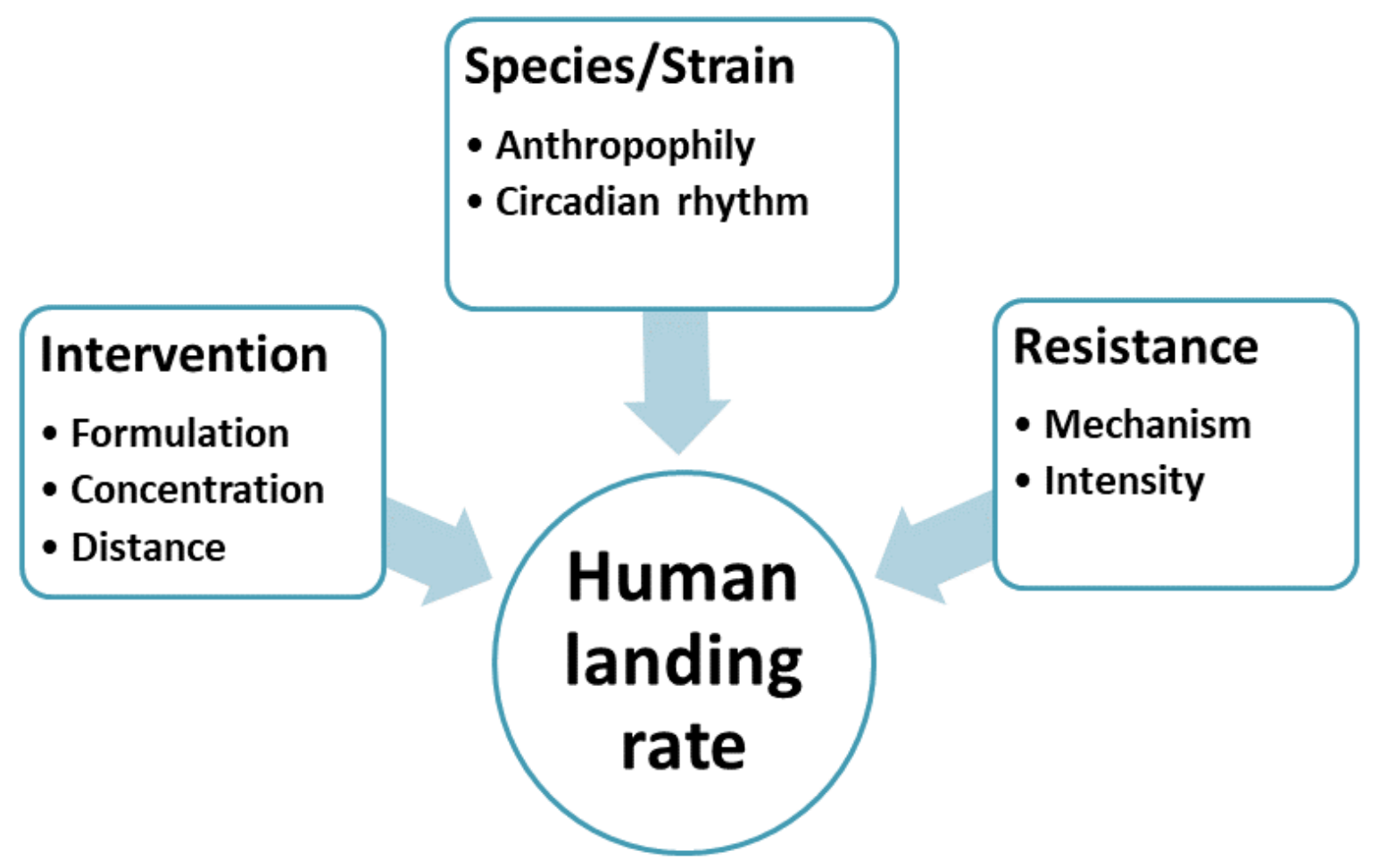

Figure 2

Factors shown to influence human landing rate and thus the protective efficacy of the EPTI 